\title{
PERFIL EPIDEMIOLÓGICO DE LOS ACCIDENTES DE TRÁNSITO EN EL PERÚ, 2005-2009
}

\author{
Víctor Choquehuanca-Vilca ${ }^{1,2, a}$, Fresia Cárdenas-García ${ }^{1,2, a}$, Joel Collazos-Carhuay,,2,c, \\ Willington Mendoza-Valladolid ${ }^{1,2, b, d}$
}

\begin{abstract}
RESUMEN
Objetivo. Determinar las características del perfil epidemiológico de los accidentes de tránsito (AT) en el Perú, en el periodo 2005 - 2009. Materiales y métodos. Estudio descriptivo, retrospectivo, se analizó fuentes secundarias de información de AT de la Policía Nacional del Perú y la Estrategia Sanitaria Nacional de Accidentes de Tránsito (ESNAT) del Ministerio de Salud. Resultados. En el quinquenio estudiado se produjeron 404120 AT, el 63,8\% de éstos sucedió en Lima. Los automóviles y las camionetas representan los tipos de vehículo más veces involucrados en estos accidentes. El exceso de velocidad y la imprudencia del conductor son las principales causas de la siniestralidad. El tipo de accidente más común es el choque (57\%). Los varones de 20 a 34 años son la población más vulnerable. Los AT han provocado 17025 muertos y 235591 lesionados en el Perú durante este periodo. Conclusiones. Los AT son un problema emergente de salud pública en el Perú. La tendencia de las cifras de mortalidad y morbilidad por accidentes de tránsito en el Perú ha seguido una curva ascendente entre los años 2005 y 2008; sin embargo, estas cifras durante el 2009 se han reducido de manera muy poco significativa. Los sistemas de información deben integrarse para proporcionar información confiable a las instituciones nacionales involucradas en esta problemática, de manera que sirvan de referencia para futuras decisiones políticas.
\end{abstract}

Palabras clave: Accidentes de Tránsito; Sistemas de información; Perfil de salud; Epidemiología; Perú (fuente: DeCS BIREME).

\section{EPIDEMIOLOGICAL PROFILE OF ROAD TRAFFIC ACCIDENTS IN PERU, 2005-2009}

\begin{abstract}
Objective. To determine the characteristics and epidemiological profile of road traffic injuries (RTI) in Peru during the period 2005-2009. Materials and methods. Descriptive, retrospective study, secondary sources of information on RTI from the National Police of Peru and the National Sanitary Strategy for the Control of Traffic Accidents (ESNAT) of the Ministry of Health were analyzed. Results. During the 5 years of study, there were 404120 TA, of which $63.8 \%$ occurred in Lima. Cars and vans are the types of vehicle most frequently involved in these accidents. Speed excess and driver's senselessness are the main causes of accidents. The most common type of accident is the crash (57\%). Males from 20 to 34 years old are the most vulnerable population. Conclusions. The RTI are an emerging public health problem in Peru. The trend of the mortality and morbidity rates due to traffic accidents in Peru has followed an increasing curve between the years 2005 and 2008; however these numbers during 2009 have been decreasing but not really significantly. Information systems must be integrated in order to give reliable information to the national institutions involved in these problematic, so that it can be used as a reference for future political decisions.
\end{abstract}

Key words: Accidents, traffic; Information systems; Health profile; Epidemiology; Peru (source: MeSH NLM).

\section{INTRODUCCIÓN}

A medida que disminuyen las causas de muerte debidas a situaciones carenciales o infecciosas, los accidentes emergen como un factor de muerte, discapacidad y enfermedad que requiere ser reducido y evitado ${ }^{(1)}$. Se tiene conocimiento que cada año millones de personas mueren por lesiones, principalmente ocasionadas por accidentes de tránsito producidas por vehículos automotores ${ }^{(2)}$.

Un accidente de tránsito (AT) es definido como una colisión en la que participa al menos un vehículo en movimiento por un camino público o privado y que

Oficina General de Defensa Nacional, Ministerio de Salud. Lima, Perú.
Estrategia Sanitaria Nacional de Accidentes de Tránsito, Ministerio de Salud. Lima, Perú.
Médico Pediatra; ${ }^{\circ}$ Médico Epidemiólogo; ${ }^{\circ}$ Cirujano Dentista; ${ }^{\text {}}$ Licenciado en Enfermería.

Recibido: 05-05-10 Aprobado: 09-06-10 
deja al menos una persona herida o muerta ${ }^{(3)}$. Estos eventos son considerados un problema emergente de salud pública y shocks microeconómicos que afectan directamente el presupuesto familiar variando la condición de pobreza de la familia afectada ${ }^{(2,4)}$.

Se calcula que cada año mueren más de 1,2 millones de personas en las carreteras a nivel mundial, $90 \%$ de las muertes se concentran en los países de bajos y medianos ingresos, quienes cuentan con menos de la mitad del parque vehicular mundial; asimismo entre 20 y 50 millones de personas padecen traumatismos no mortales. Con relación a la mortalidad por accidentes de tránsito, es conocido que alcanza sus máximas cifras en la población económicamente activa ${ }^{(5)}$, razón suficiente para ser considerada una pandemia en buena parte del planeta $^{(6)}$.

En el Perú, entre los años 1999 y 2008 se han registrado 779141 AT, los cuales han ido incrementándose año tras año a partir del año 2002 a la fecha ${ }^{(7)}$. El Ministerio de Salud notificó que en el año 2008 se hospitalizaron 47214 personas con lesiones atribuibles a AT, gran porcentaje de ellas pertenece al grupo etario entre 20 a 34 años; además, se estima que unas 117900 personas quedaron con algún grado de discapacidad entre los años 2005 y 2008. Estas cifras han conllevado a que en nuestro país los AT representen la causa de mayor carga de enfermedad ${ }^{(8)}$.

En la Región Callao ${ }^{(9)}$, se describieron las características epidemiológicas de AT durante el período 1996-2004, donde se reitera que los AT son un grave problema de salud pública para la jurisdicción de estudio, registrándose 25 mil en nueve años, teniendo el $3 \%$ de estos, consecuencias fatales. El Ministerio de Salud evaluó los AT fatales producidos en el 2008 a nivel regional, encontrando que la mayor cantidad de eventos en dicho año se concentra en nuestra capital (51,3\%), además, menciona que en regiones como Amazonas, Apurímac, Ayacucho y Huancavelica, departamentos con población en extrema pobreza, las muertes por AT se han incrementado $100 \%$ con respecto al año $2007^{(7,10)}$.

En muchos países se han iniciado diversas acciones que buscan controlar y prevenir la ocurrencia de estos incidentes, observándose que en varios de ellos no resultan útiles las campañas preventivas que intentan abarcar simultáneamente a todo tipo de accidentes (11); esto debido a que las estrategias de prevención difieren considerablemente según las clases de accidentes, los sitios de ocurrencia o los grupos etarios involucrados.

Los países que han logrado disminuir en forma importante la incidencia y la mortalidad por AT han basado su estrategia, entre otras medidas, en construir un sistema de registro lo más completo y exhaustivo posible ${ }^{(12)}$, el cual cuantifica tres aspectos fundamentales: la medición de la magnitud o frecuencia relativa del fenómeno objeto de intervención, la identificación de los factores asociados con dicho fenómeno y la importancia de su asociación, así como la selección de las estrategias y acciones que hayan demostrado una probada eficacia en la modificación de los factores asociados o del propio fenómeno objeto de intervención. La metodología epidemiológica permite definir y cuantificar los tres conceptos clave antes citados ${ }^{(13)}$.

Este artículo realiza una aproximación al perfil epidemiológico de los accidentes de tránsito en los últimos cinco años en el Perú, con el propósito de que sirva de orientación para el diseño de las políticas, estrategias y acciones que contribuyan a la reducción de los daños originados por estos eventos a nivel regional y nacional.

\section{MATERIALES Y MÉTODOS}

Se realizó un estudio descriptivo retrospectivo, basado en fuentes secundarias de información de AT a nivel nacional en el periodo comprendido entre 2005 y 2009. La fase de recolección de datos se llevó a cabo entre febrero y marzo de 2010.

Las fuentes secundarias de información utilizadas para el análisis fueron las de la Dirección de Estadística del Estado Mayor de la Policía Nacional del Perú (PNP), así como las de la Estrategia Sanitaria Nacional de Accidentes de Tránsito (ESNAT) del Ministerio de Salud.

La PNP es la única institución que recolecta las estadísticas básicas sobre AT en el país; sin embargo, pese a mostrar limitaciones de información, es una fuente de referencia muy utilizada por diferentes instituciones en cuanto a estadísticas de AT se refiere ${ }^{(14)}$.

La información proporcionada por la Dirección de Estadística del Estado Mayor de la Policía Nacional del Perú comprendió la base de datos del periodo 2005 2009, en formato Excel. Esta información permitió el análisis de las siguientes variables: número de AT, tipos de vehículos involucrados, tipo y causas de accidentes, día de la semana y horario en que se producen, edad y sexo de los involucrados y número de accidentes fatales y no fatales.

La ESNAT, fue creada por el Ministerio de Salud, por considerar a los AT como un problema de salud pública 
de gran impacto socioeconómico, constituyéndose en la respuesta del sector salud a esta problemática. La fuente de datos con la que cuenta la ESNAT es producto de los consolidados estadísticos nacionales proporcionados por la Oficina General de Estadística e Informática (OGEI), la Dirección General de Epidemiología (DGE) y el Instituto Nacional de Rehabilitación (miembros del Comité Técnico Permanente de la ESNAT). La información derivada de la OGEI es producto de los datos ingresados a nivel nacional a través de la Hoja HIS correspondientes a los años 2005-2008; los datos reportados por la Dirección General de Epidemiología son resultado del consolidado de la Ficha de Vigilancia Epidemiológica de Accidente de Tránsito (2007-2009).

En cuanto a la consistencia de los datos, la información proporcionada por la PNP comprende el periodo de estudio; sin embargo, presenta limitaciones importantes como la referente a la diferenciación por grupo etario, pues la información reportada por la PNP sólo distingue mayores y menores de 18 años de edad. De igual modo, existen variables muy ambiguas como en el caso de las causas de AT, donde la variable "imprudencia del conductor" no posee una clara definición, esta variable podría involucrar otras como exceso de velocidad o ebriedad del conductor.

La información proporcionada por la ESNAT comprende de manera parcial el periodo del estudio, debido a que la información sólo se encuentra procesada hasta el 2008 para el caso de la OGEI, y el llenado de la Ficha de Vigilancia Epidemiológica recién se empezó a realizar a partir del año 2007, para el caso de la DGE.

Con las fuentes secundarias de información mencionadas se realizó al cálculo de estadísticas vitales como la incidencia, mortalidad y letalidad. La incidencia estuvo determinada por el número de casos nuevos de muertes y heridos por AT que se dieron durante el período determinado por el estudio. De igual modo, la mortalidad se calculó dividiendo el número de personas muertas entre el total de población susceptible estimada, según el Instituto Nacional de Estadística e Informática por 100000 habitantes. También se realizó el cálculo de la tasa de mortalidad por parque automotor (número de muertes por AT/parque automotor del año) por 10000 vehículos. La letalidad se obtiene de dividir el número de muertes sobre el número de persona con daños a la salud productos por AT por 100 .

Se usaron para el análisis medidas de posición y dispersión como herramientas de estadística descriptiva; asimismo, los análisis estadísticos reportaron frecuencias y proporciones. Se hizo uso del programa estadístico SPSS versión 13 y Excel 2007.

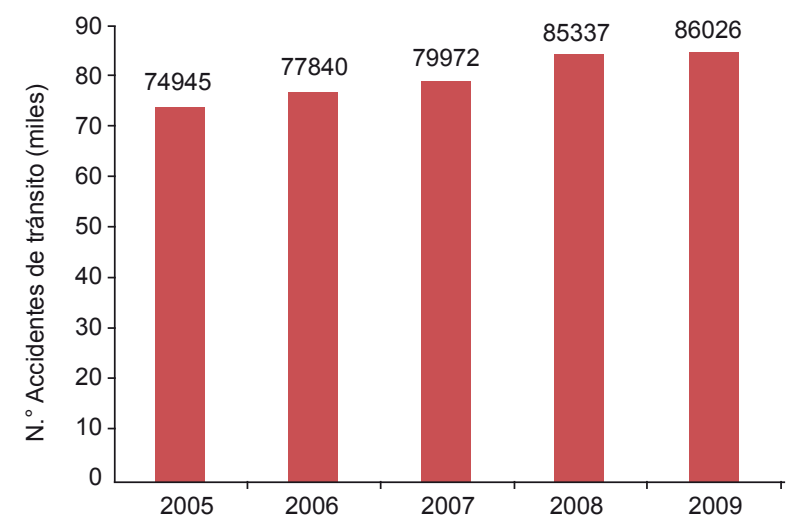

Figura 1. Frecuencia de accidentes de tránsito en el Perú, 2005-2009

Fuente: Dirección de Estadística del Estado Mayor de la Policía Nacional del Perú.

\section{RESULTADOS}

Según la Dirección de Estadística del Estado Mayor de la Policía Nacional del Perú, durante los últimos cinco años, se han producido 404120 AT a nivel nacional. En la Figura 1, la tendencia de los AT en nuestro país muestra un incremento constante, registrándose en el año 2009, la mayor cifra de AT de los últimos cinco años (86 026 accidentes).

El tipo de accidente más común lo constituye el choque; evento que ha representado en los últimos cinco años el $57,8 \%$ de todos los accidentes acontecidos, incrementando su frecuencia de manera ascendente año tras año. Por otro lado, los atropellos representan el $27,7 \%$ del total de accidentes; disminuyendo su frecuencia sólo durante el último año del estudio.

El 35,9\% de los AT suceden entre los días viernes y sábado, siendo el domingo, el día donde menor siniestralidad se registra $(12,1 \%)$. En cuanto al horario de ocurrencia, los AT se producen mayormente entre las dos de la tarde y las ocho de la noche $(30,0 \%)$.

El tipo de vehículo más involucrado en los accidentes tránsito es el automóvil (40,3\%), las camionetas $(21,8 \%)$ y las unidades denominadas "mototaxi" (10\%). Es necesario indicar que dentro del rubro "camionetas" están comprendidas las denominadas "combis".

La tendencia de los accidentes producidos por automóviles ha presentado durante los últimos cinco años una conducta creciente; con respecto a las camionetas, la siniestralidad de estas se ha mantenido constante; sin embargo, son las "mototaxis" quienes muestran una predisposición a incrementarse de manera considerable conforme transcurren los años. 


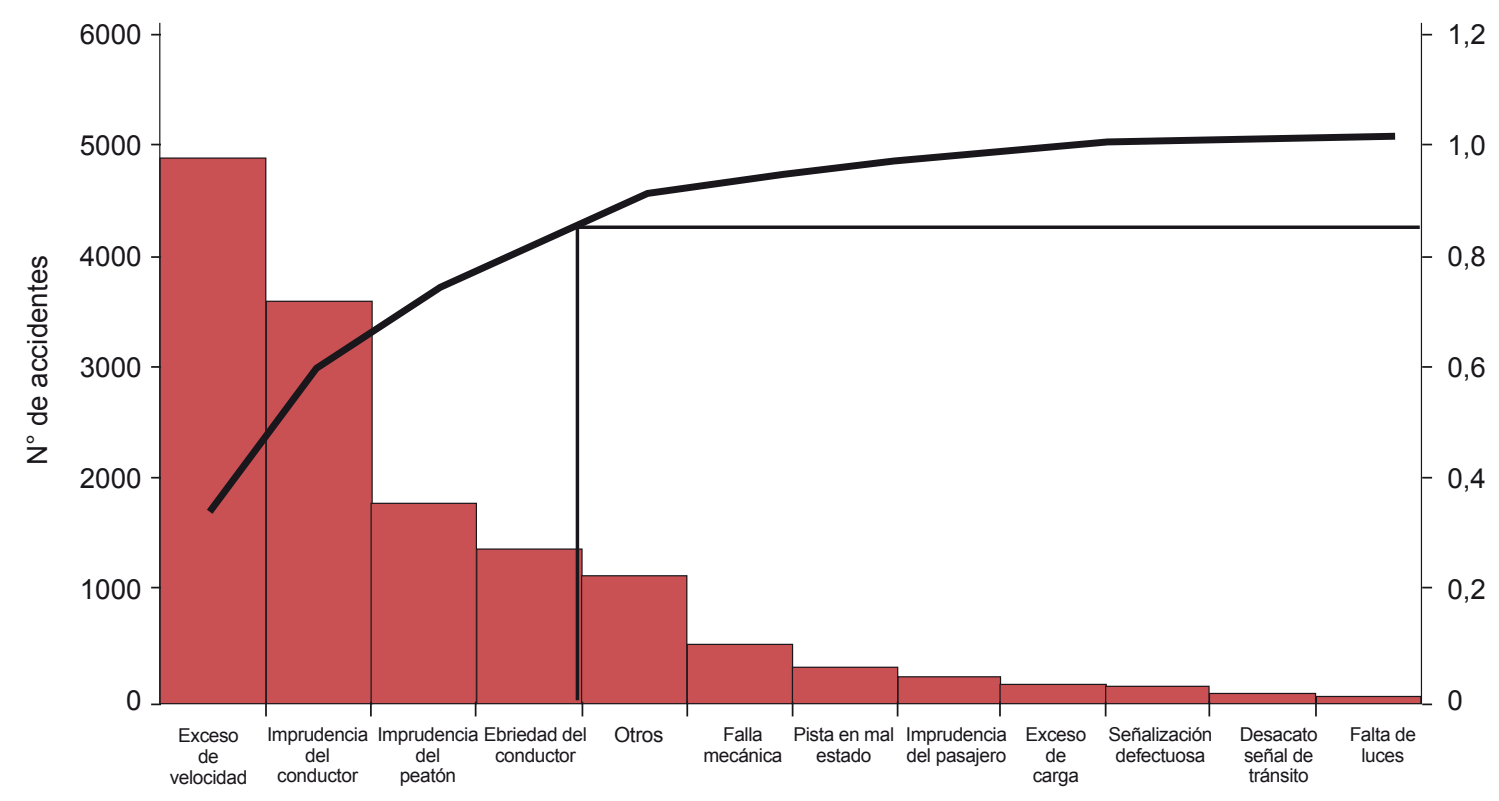

Figura 2. Causas de los accidentes de tránsito en el Perú, 2005-2009.

Fuente: Dirección de Estadística del Estado Mayor de la Policía Nacional del Perú.

El exceso de velocidad es la primera causa de accidentes de tráfico en nuestro país $(30,8 \%)$, seguido de la imprudencia del conductor $(26,1 \%)$, otras causas $(12,2 \%)$ y ebriedad del conductor $(9,6 \%)$. Si bien los accidentes producidos por el exceso de velocidad han disminuido en el año 2009, los producidos por el consumo de alcohol y la imprudencia del conductor han seguido manteniendo una conducta creciente en los últimos cinco años.

A nivel nacional entre los años 2005 y 2009, los AT se han concentrado mayormente en las regiones de Lima $(63,8 \%)$, Arequipa, La Libertad y Callao con $5,8 \% ; 4,8 \%$ y $3,7 \%$ respectivamente.

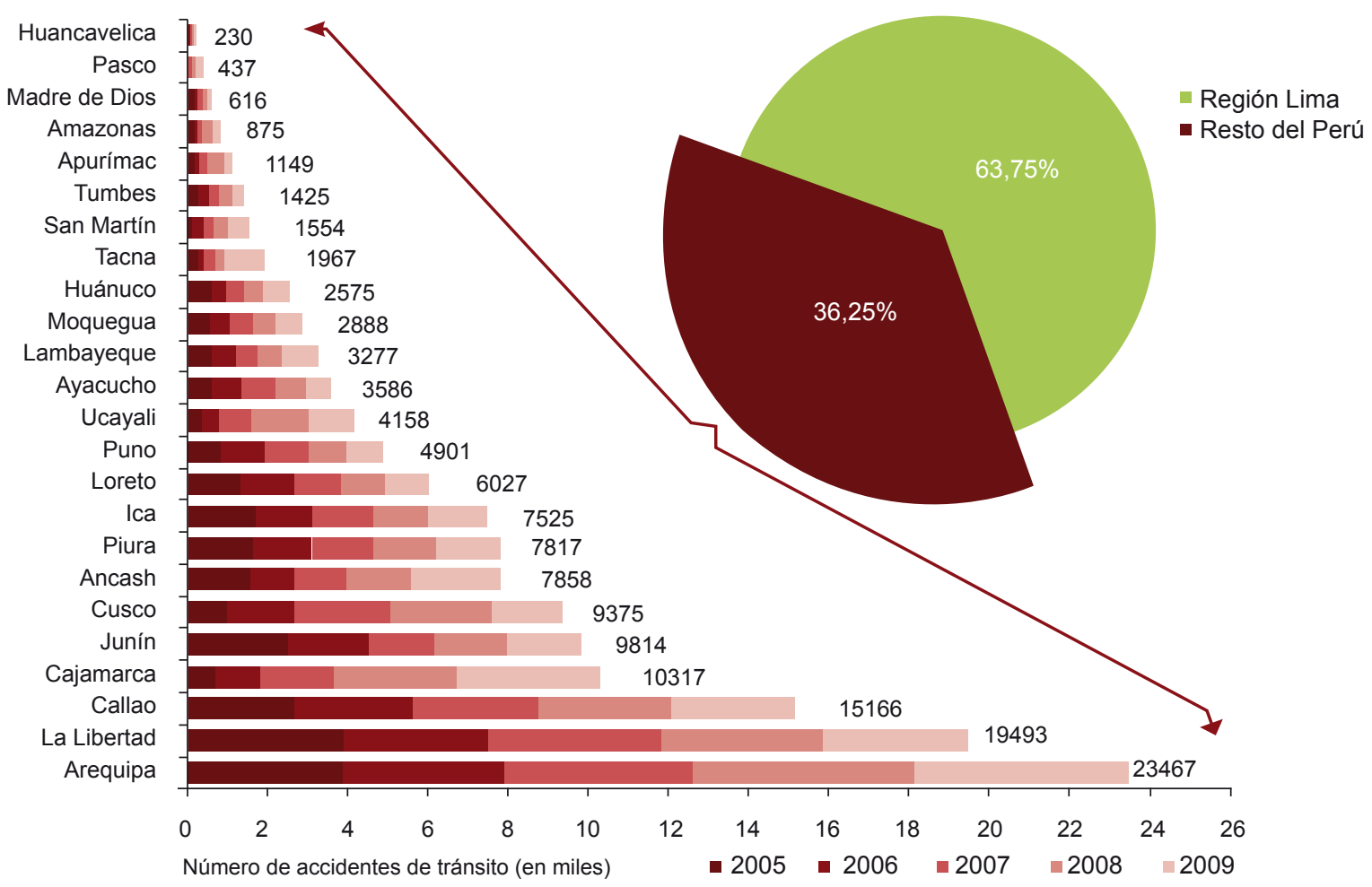

Figura 3. Accidentes de tránsito por regiones en el Perú, 2005-2009.

Fuente: Dirección de Estadística del Estado Mayor de la Policía Nacional del Perú. 


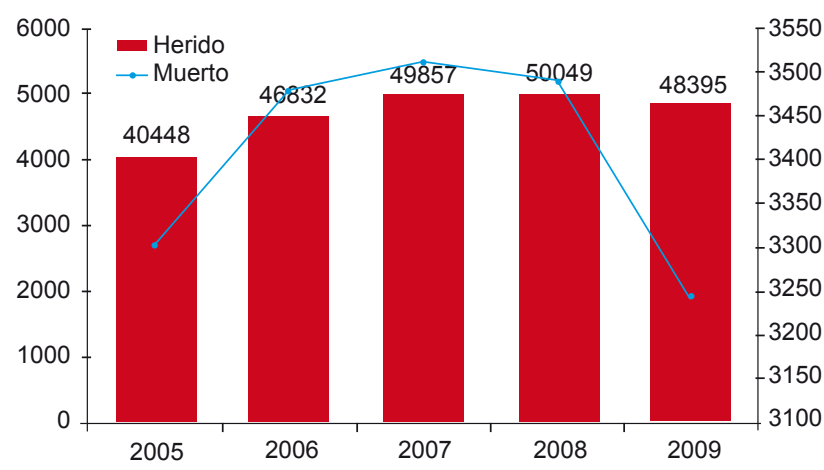

Figura 4. Muertos y heridos en accidentes de tránsito en el Perú, 2005-2009.

Fuente: Dirección de Estadística del Estado Mayor de la Policía Nacional del Perú.

En la región Lima, la frecuencia de AT mantiene una curva ascendente continua, obteniéndose en el año 2009 la cifra más alta de los últimos diez años (52 916 AT). De igual modo, las regiones de Arequipa, La Libertad y Callao han mostrado un incremento evolutivo de accidentes desde el año 2005 al 2008; sin embargo, en el año 2009 la curva de frecuencia evidencia una ligera tendencia hacia el descenso. Casos particulares suceden en las regiones de Ancash, Pasco y Tacna, donde el año 2009 han aumentando considerablemente los AT.

El parque automotor del país ha crecido, en los últimos años, de manera exponencial, así como también de manera muy desordenada, hasta el año 2009 se habían registrado un total de 1695003 vehículos, cifra que va acorde con la ocurrencia de los AT.

\section{MORTALIDAD POR LOS ACCIDENTES DE TRÁNSITO}

En cuanto a mortalidad se refiere, entre los años 2005 y 2009 los AT han ocasionado 17025 muertes a nivel nacional. El año 2007 se registró la mayor cantidad de muertes en el país (3510); asimismo, es necesario señalar la disminución de éstas en los dos últimos años (Figura 4).

En todo el país, las muertes por AT son consecuencias de atropellos $(47,2 \%)$ y choques $(32.0 \%)$. El $46,8 \%$ de estas muertes, han sido provocadas por automóviles y camionetas. Las principales causas de mortalidad en los AT son atribuidas al exceso de velocidad $(33,82 \%)$; la imprudencia del conductor $(24,82 \%)$ y la imprudencia del peatón (12,58\%). Las muertes por AT se producen mayormente entre los días viernes y domingo $(52 \%)$ entre dos de la tarde y ocho de la noche $(32,4 \%)$.

En lo que concierne a la tasa de mortalidad por AT, en nuestro país esta ha mostrado una ligera curva descendente desde el año 2006 hasta el final del

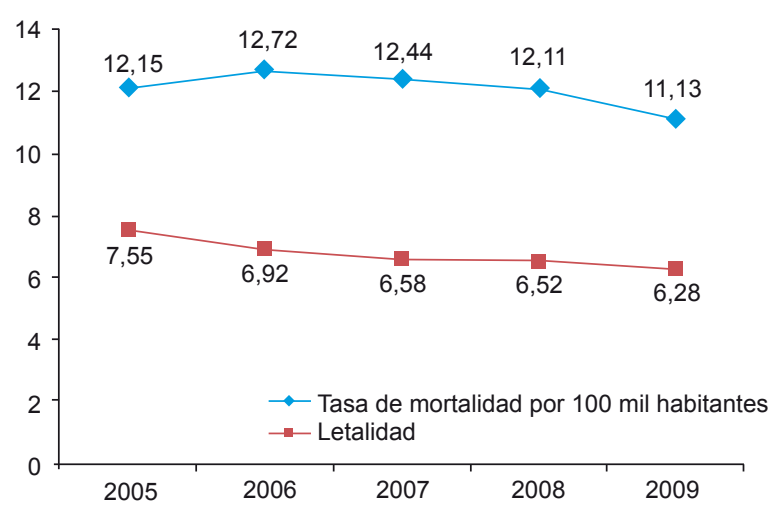

Figura 5. Tasa de mortalidad y letalidad por accidentes de tránsito, Perú 2005-2009.

Fuente: Dirección de Estadística del Estado Mayor de la Policía Nacional del Perú.

2009. Observando la Figura 5, podemos evidenciar la tendencia descendente descrita anteriormente.

De igual modo, en la Figura 6, se representan la tendencia de las tasas de mortalidad por 10000 vehículos, la cual se obtuvo dividiendo el número de muertes sobre el parque vehicular por año.

Del total de muertes acontecidas como consecuencia de los AT, el $79 \%$ corresponde al sexo masculino. El grupo etario más afectado resultó ser el comprendido entre 20 y 34 años. Las regiones con los más altos registros de muerte son: Lima (28,3\%); Cusco (10,8\%); La Libertad (10,3\%); Puno (6,5\%) y Ancash $(5,7 \%)$ respectivamente.

En el 2009 las regiones de Madre de Dios (3,8), Cusco $(3,5)$, y Amazonas $(3,2)$ tuvieron las más altas tasas de mortalidad por AT en nuestro país; por otro lado, las

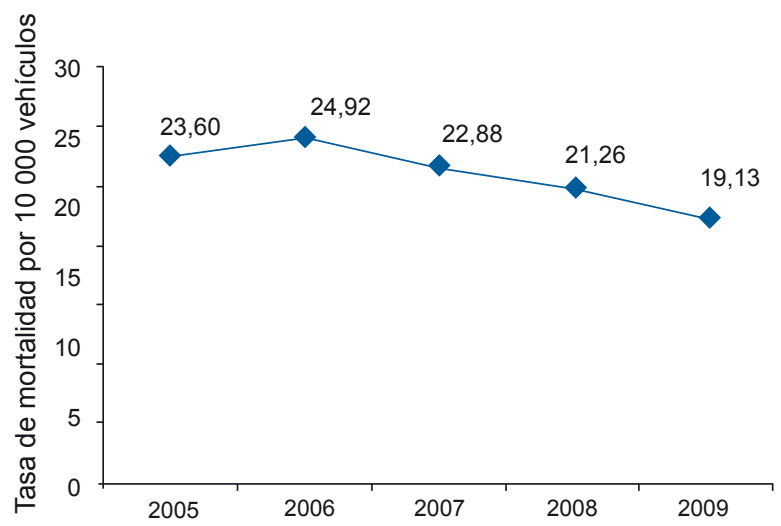

Figura 6. Tasa de mortalidad por 10000 vehículos, Perú 20052009.

Fuente: Dirección de Estadística del Estado Mayor de la Policía Nacional del Perú y Gerencia de Transporte Terrestre del Ministerio de Transportes y Comunicaciones. 
regiones de Huánuco $(0,4)$, Callao $(0,3)$ y Cajamarca $(0,3)$ presentan la tasas de mortalidad más bajas.

Las tasas de letalidad por AT, muestran una discreta disminución a lo largo del período del estudio. Las regiones de la selva y la sierra poseen las mayores tasas de letalidad en nuestro país. Madre de Dios $(32,84)$, Puno $(27,16)$ y Amazonas $(24,63)$ poseen las tasas más altas mientras que Lima y Callao ocupan los últimos lugares en cuanto a tasas de letalidad se refiere.

\section{MORBILIDAD POR LOS ACCIDENTES DE TRÁNSITO}

En los últimos cinco años los AT han lesionado a 233718 personas a nivel nacional, En el año 2008 se registró la cifra más alta de lesionados 50059 de los últimos tiempos. Al igual que la mortalidad, la morbilidad por AT ha mostrado una ligera disminución en el año 2009.

La mayor parte de las lesiones ocasionadas por los AT son consecuencias de choques $(58,7 \%)$ y atropellos (27\%). Los automóviles $(40,1 \%)$ y las camionetas $(21,8 \%)$ han sido los tipos de vehículos mayormente involucrados en lesiones por AT.

Las principales causas de morbilidad en los AT son atribuidas al exceso de velocidad (30,6\%); la imprudencia del conductor $(26,1 \%)$ y la ebriedad del conductor $(9,6 \%)$.

Los AT no fatales se producen mayormente entre los días viernes y domingo $(47,1 \%)$ entre las ocho de la mañana y las dos de la tarde $(30,0 \%)$. Finalmente, de cada diez personas que sufren lesiones por AT, siete corresponden al sexo masculino.

Las regiones que más reportan lesionados, producto de los AT, son Lima (43,5\%), seguidos de Callao, Arequipa y La Libertad con 12,$8 ; 6,0$ y $4,9 \%$ respectivamente.

\section{DISCUSIÓN}

Los AT constituyen un problema emergente de salud pública en los países en vías de desarrollo, donde la morbilidad y mortalidad van de la mano con el incremento desmesurado del parque automotor y una desordenada urbanización (15-17).

Según el Informe sobre la situación de la seguridad vial de la Organización Mundial de la Salud, publicado en agosto de 2009, el Perú posee la segunda tasa más alta de mortalidad por cada 100000 habitantes a nivel de Sudamérica, pese a contar con el parque automotor más bajo en América Latina ${ }^{(5)}$.
En el Perú, durante el período 2005 y 2009, se han producido 404120 AT, incrementándose estas cifras en $14,79 \%$ en los últimos cinco años; de igual manera, es prudente tener en cuenta que el parque automotor también ha sufrido un incremento de $17,71 \%$ en el mismo periodo.

La distribución a nivel nacional de los AT ubica a Lima como la región que concentra la mayor cantidad de estos eventos $(63,8 \%)$, seguidamente Arequipa, La Libertad y Callao completan la lista de regiones con mayor cantidad de AT, lo que llama la atención es que no siempre la región que posee el mayor parque automotor tiene la mayor cantidad de accidentes como en el caso de Arequipa, que pese a contar con el tercer mayor parque automotor, sólo detrás de La Libertad, se ubica como la segunda región con mayor siniestralidad.

Según los datos analizados, en los últimos cinco años, han muerto en las pistas del Perú 17025 personas, producto en su mayoría por atropellos de automóviles y camionetas; la región de Lima es la que mayor muertes registra por AT, situación que parece obvia al tener en cuenta que es la región que mayor parque automotor posee; sin embargo, al hacer un análisis del año 2009 en razón de las tasas de mortalidad por 100 vehículos, encontramos que son las regiones de Amazonas $(5,8)$, Madre de $\operatorname{Dios}(4,7)$ y Huancavelica $(3,5)$ las que mayor tasa de mortalidad poseen; por el contrario, Moquegua $(0,2)$, Tacna $(0,1)$ y Lima $(0,1)$ poseen las tasas de mortalidad más bajas a nivel nacional, situación que se confirma cuando se analiza la tasa de mortalidad por 10000 habitantes, donde las regiones de Madre de Dios $(2,8)$ Cusco $(3,5)$, Amazonas $(3,2)$, Puno $(2,2)$ y La Libertad $(1,9)$ presentan las más altas tasas, esto podría explicarse por el desarrollo de accidentes masivos por ómnibus interprovinciales, sucedidos de manera cotidiana en el interior del país y la inaccesibilidad geográfica donde se desarrollan estos accidentes, de manera que resulta dificultoso prestar alguna atención inicial de salud. Es importante tener en cuenta que un accidente provocado por un ómnibus interprovincial causa 2,63 veces más heridos y 2,33 más muertos que el resto de unidades.

Es preciso comentar que carecemos de un sistema organizado de atención prehospitalaria que responda de la manera eficiente ante los AT ${ }^{(17,18)}$, es una realidad innegable que los primeros auxilios frente a este tipo de eventos son brindados, por lo general, por las personas que se encuentran alrededor, estén o no involucradas, realizando mucha veces maniobras que provocan un mayor daño a la salud. Esta situación puede fácilmente explicar por qué las mayores tasas de letalidad por AT se encuentran en las regiones de la selva y la sierra, donde la agreste geografía impide que la atención de salud pueda llegar a los afectados. 
Los AT clasificados como "choques" por las fuentes de información revisadas, constituyen el $57,79 \%$ del total de accidentes en los últimos cinco años, pese a ser la mayoría de tipo de accidentes involucrados en estos eventos, cuando se hace la revisión de la mortalidad estos son desplazado por los "atropellos" que ocasionan la mayoría de muertes $(47,15 \%)$, dada la vulnerabilidad del cuerpo humano frente a un vehículo.

El exceso de velocidad y la imprudencia del conductor representan las principales causas de los AT en nuestro país, el consumo de alcohol como causa de los AT se ubica en el tercer lugar, sin embargo la tendencia de esta variable a lo largo del estudio ha ido incrementando progresivamente año tras año.

El parque automotor en nuestro país ha ido incrementándose a lo largo de los últimos cinco años, actualmente el parque automotor peruano cuenta con casi un millón setecientos mil vehículos automotores (1 695 004), encontrándose muy por debajo del promedio de vehículos a nivel de Sudamérica (promedio: 7000 000); sin embargo, pese a la cantidad, es un factor muy importante a considerar, ya que gran parte de este parque automotor, sobre todo el de transporte interprovincial, no aprueba las revisiones técnicas dispuestas; la problemática se agudiza aun más cuando adicionamos a esta la importación de vehículos usados y de aquellos que brindan poca seguridad ("mototaxis", vehículos modelo "tico" entre otros). Los automóviles y las camionetas son los vehículos comprometidos con mayor frecuencia en los AT fatales y no fatales a nivel nacional, a diferencia de regiones amazónicas, donde los vehículos motorizados de tres ruedas (llamados "mototaxis") son los que cuentan con mayor frecuencia de AT fatales.

En el Perú los AT ocurren generalmente entre los días viernes y sábado en el intervalo de las dos de la tarde y las ocho de la noche, cifras que coinciden con las costumbres de la población relacionadas con la diversión, ocio y al alto tránsito y congestión vehicular ${ }^{(18,19)}$.

La población joven comprendida entre los 20 y 34 años es el grupo etario más afectado por los AT, resultado que coincide con las estadísticas mundiales revisadas al respecto; como podemos evidenciar es la población económicamente activa la más afectada por estos eventos, limitando el progreso de la familia de las víctimas quienes tienen que asumir el costo económico y psicológico de las consecuencias de las lesiones o las muertes que se puedan producir por los AT.

Pese a mostrar las tasas de mortalidad, morbilidad y letalidad una conducta decreciente en los últimos años, esta realidad contrasta con la que a diario se evidencia en los diferentes medios de comunicación del país, lo que nos permite reafirmar las limitaciones ya descritas de estas fuentes secundarias de información.

Este artículo espera contribuir al desarrollo de mayores conocimientos y evidencias sobre la situación actual de la problemática de los accidentes de tránsito en nuestro país, de manera que esta información facilite tanto la identificación de áreas prioritarias, como la adecuada toma de decisiones por parte de las instituciones comprendidas en el tema.

\section{AGRADECIMIENTOS}

Al Licenciado William Anchiraico Agudo, de la Oficina General de Estadística e Informática del Ministerio de Salud, por su colaboración en el análisis de los resultados del presente artículo. A la Dirección de Estadística del Estado Mayor de la Policía Nacional del Perú y la Dirección General de Epidemiología del Ministerio de Salud por el acceso a la información que recolectan.

\section{Fuente de Financiamiento}

Autofinanciado.

\section{Conflictos de Interés}

Los autores declaran no tener ningún conflicto de interés en la publicación de este artículo.

\section{REFERENCIAS BIBLIOGRÁFICAS}

1. Ameratunga $\mathbf{S}$, Hijar $\mathbf{M}$, Norton R. Road-traffic injuries: confronting disparities to address a global health problem. Lancet. 2006; 367(9521): 1533-40.

2. Organización Mundial de la Salud. Informe mundial sobre prevención de los traumatismos causados por el tránsito. Ginebra: OMS; 2004.

3. Perú, Ministerio de Salud. Norma técnica de salud para la vigilancia epidemiológica de lesiones por accidentes de tránsito. Lima: MINSA; 2007.

4. Bambaren C. Características epidemiológicas y económicas de los casos de accidentes de tránsito atendidos en el Hospital Nacional Cayetano Heredia. Rev Med Hered. 2004; 15:30-36.

5. Organización Mundial de la Salud. Informe sobre la situación mundial de la seguridad vial. Es hora de pasar a la acción. Ginebra: OMS; 2009.

6. Mohan D. Road traffic injuries—a neglected pandemic. Bull World Health Organ. 2003; 81(9): 684-85.

7. Perú, Ministerio de Salud. Accidentes de Tránsito Problema de Salud Pública Informe Nacional. Lima: MINSA, 2009.

8. Velásquez A. La carga de enfermedad y lesiones en el Perú y las prioridades del plan esencial de aseguramiento universal. Rev Peru Med Exp Salud Publica. 2009; 26(2): 222-31. 
9. Wong P, Salazar D, Bérninzon L, Rodríguez A, Salazar $\mathbf{M}$, Valderrama $\mathbf{H}$, et al. Caracterización de los accidentes de tránsito en la región Callao-Perú, 1996-2004. Rev Peru Epidemiol. 2009; 13(3): e3.

10. Perú, Ministerio de Salud. Plan Nacional de la Estrategia Sanitaria Nacional de Accidentes de Tránsito 2009-2012. Lima: MINSA 2009.

11. Vásquez R. Causas de los accidentes de tránsito desde una visión de la medicina social. El binomio alcohol-tránsito. Rev Med Uruguay. $2004 ; 20(3): 178-86$.

12. Waisman I, Nuñez J, Sanchez J. Epidemiología de los accidentes en la infancia en la Región Centro Cuyo. Rev Chil Pediatr. 2002: 73(4): 404-14.

13. Kemp A, Sibert J. Childhood accidents: epidemiology, trends and prevention. J Accid Emerg Med. 1997; 14(5): 316-20.

14. Alfaro-Basso D. Problemática sanitaria y social de la accidentalidad del transporte terrestre. Rev Peru Med Exp Salud Publica. 2008; 25(1): 133-37.

15. Söderlund N, Zwi AB. Mortalidad por accidentes de tránsito en países industrializados y en desarrollo. Bol Oficina Sanit Panam. 1995; 119(6): 471-80.
16. Garg N, Hyder AA. Exploring the relationship between development and road traffc injuries: a study from India. Eur J Public Health. 2006; 16(5): 487-91.

17. Defensoría del Pueblo. Informe Defensorial $N^{\circ} 108$. Pasajeros en riesgo: La seguridad en el transporte interprovincial. Lima: Defensoría del Pueblo 2008.

18. García F, Cieza J, Alvarado B. Características de las atenciones registradas por la policía en el servicio de emergencia de un hospital de Lima, 2001. Rev Peru Med Exp Salud Publica. 2005; 22(1): 71-75.

19. Rey de Castro J, Rosales E, Egoavil M. Somnolencia y cansancio durante la conducción: accidentes de tránsito en las carreteras del Perú. Acta Med Peru. 2009: 26(1): 48-54.

\section{Correspondencia: Dr. Willington Abraham Mendoza Valladolid \\ Dirección: Calle Marconi 317 - San Isidro, Lima, Perú. \\ Teléfono: (511)997-440-458 / (511)999-408-390 \\ Correo electrónico: abraham69@voila.fr; wmendozav@minsa. gob.pe}

\section{Suscríbete en forma electrónica y gratuita a los contenidos de la}

Revista Peruana de Medicina Experimental y Salud Pública, ingresa a WWW.ins.gob.pe, selecciona el icono de la revista y envíanos tus datos.

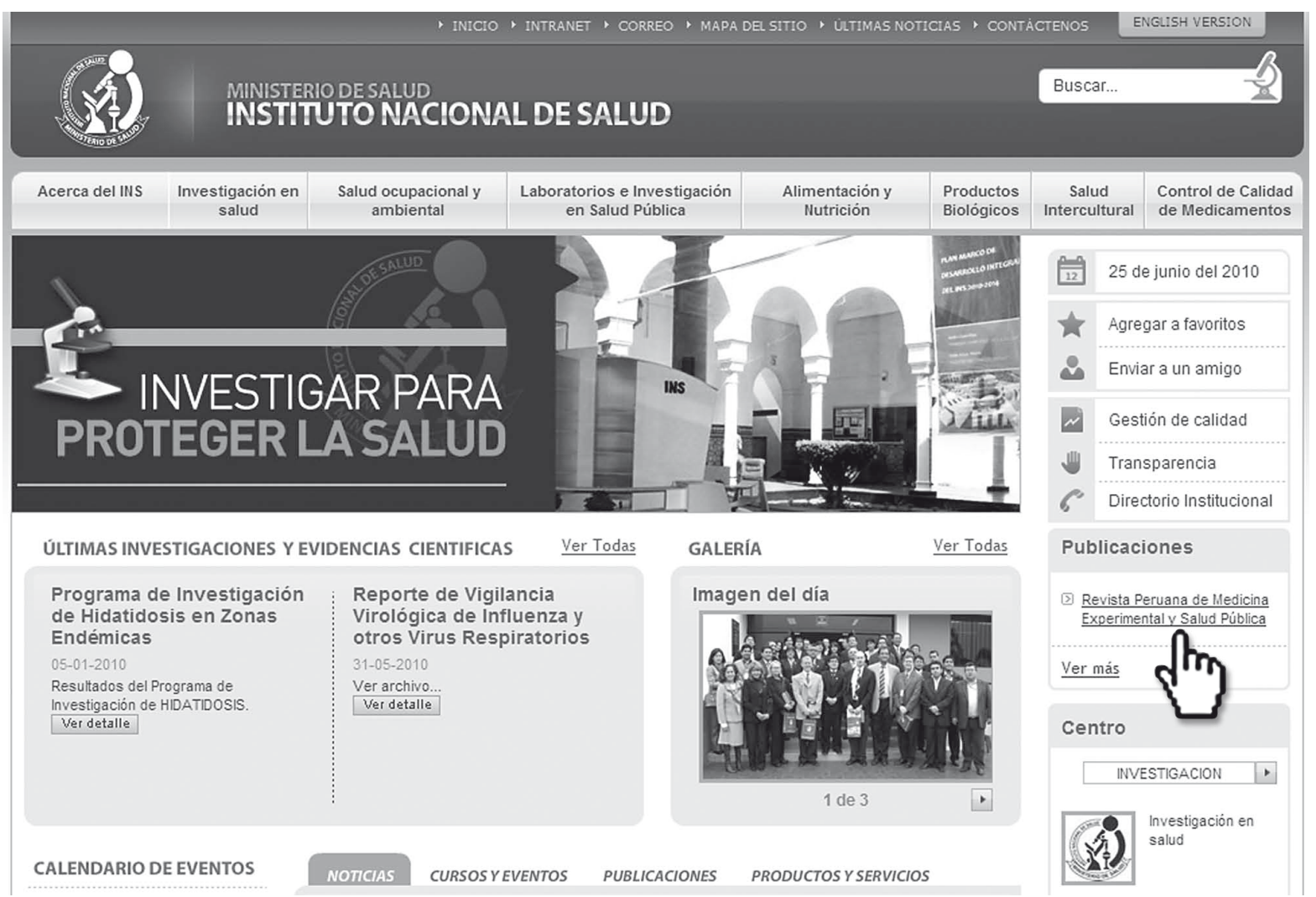

